\title{
1 Co-evolved maternal effects selectively eliminate offspring depending on resource availability
}

2

3 Bin-Yan Hsu ${ }^{1,2^{*}}$, Martina S. Müller ${ }^{1,3}$, Christoph L. Gahr ${ }^{1,4}$, Cor Dijkstra ${ }^{1 \dagger}$, Ton G. G. G. Groothuis ${ }^{1}$

$4{ }^{1}$ Behavioural Biology, Groningen Institute for Evolutionary Life Sciences, University of Groningen,

$5 \quad$ Nijenborgh 7, 9747 AG, Groningen, the Netherlands

$6 \quad{ }^{2}$ Department of Biology, University of Turku, 20014, Turku, Finland

$7{ }^{3}$ Department of Natural Resource Sciences, University of Rhode Island, Kingston, Rhode Island,

8 02881, United States

$9{ }^{4}$ Department of Evolutionary Ecology, Max Planck Institute for Evolutionary Biology, 24306 Plön,

10 Germany

$11 *$ Corresponding author: biyahs@utu.fi

$12 \dagger$ deceased 


\section{Abstract}

14 Many plants and animals adaptively downsize the number of already-produced propagules if

15 resources become insufficient to raise all of them. In birds, mothers often induce hatching asynchrony

16 by incubating first eggs before last eggs are laid, creating an age/size hierarchy within broods which

17 selectively eliminates the smallest chicks in poor food conditions. However, mothers also deposit

18 more testosterone into late-laid eggs, which boosts competitive abilities of younger chicks,

19 counteracts the competitive hierarchy, and ostensibly creates a paradox. Since testosterone also carries

20 costs, we hypothesized that benefits of maternally deposited testosterone outweigh its costs in good

21 food conditions, but that testosterone has a net detrimental effect in poor food conditions. We found

22 experimental evidence that elevated maternal testosterone in the egg caused higher chick mortality in

23 poor food conditions but better chick growth in good food conditions. These context-dependent

24 effects resolve the paradox, suggesting co-evolution of two maternal effects, and explain inconsistent

25 results of egg hormone manipulations in the literature.

\section{Keywords}

28 Food conditions, Hatching asynchrony, Maternal effects, Maternal testosterone, Sibling competition 


\section{Introduction}

Throughout the plant and animal kingdoms, mothers routinely overproduce propagules, and competition between them culls superfluous offspring until family size matches available resources $^{1,2}$, thereby neatly (albeit harshly) resolving the evolutionary trade-off between offspring quantity and quality. The severity of resource limitation determines the intensity of propagule competition; when food is scarce, siblings die, e.g. by siblicide in spotted hyena broods ${ }^{3,4}$ and blue-footed booby chicks ${ }^{5}$, intra-fruit seed abortion in plants ${ }^{6}$, maternal crushing and/or starvation in piglets ${ }^{7,8}$, or sibling cannibalism in ladybird beetles ${ }^{9}$. Mothers can improve the efficiency with which sibling rivalry eliminates offspring by creating age/size hierarchies among their young that make some siblings less able to compete than others, allowing efficient culling of their numbers when needed. For example, in birds, mothers induce hatching asynchrony by initiating incubation before all eggs have been laid.

This causes chicks from later-laid eggs to have a later start on development, to hatch later, and therefore to be smaller and weaker when competing with their older siblings for parental food provisioning.

Mothers also employ other tools for creating competitive asymmetries, including furnishing their embryos with maternal hormones ${ }^{10}$. Such hormone-mediated maternal effects have substantial and long-lasting impacts on the development of morphology, brain and behaviour ${ }^{10,11}$ and are important in plants ${ }^{12}$, insects ${ }^{13}$, reptiles ${ }^{14}$, fish $^{15}$ and other vertebrates ${ }^{10,16}$. For example, in spotted hyenas, cubs from high-ranked mothers are exposed prenatally to higher levels of maternal androgens via the placenta, which makes them more aggressive after birth ${ }^{17}$. In birds, maternal androgens, like testosterone (a potent sex steroid hormone) are deposited into eggs in substantial quantities that vary systematically with environmental conditions and also according to the position of the egg in the laying sequence in a given breeding attempt (i.e. clutch) ${ }^{18,19}$. Because in most bird species, later-laid eggs contain higher concentrations of maternal androgens, the hatching asynchrony adjustment hypothesis $(\mathrm{HAAH})^{18,19}$ proposes that this increased deposition of maternal androgens in late-laid eggs functions as a compensation for the competitive disadvantage of the chicks from these eggs. Indeed, experimental manipulation of egg androgens, especially testosterone, has revealed that these 
56 hormones stimulate the competitive ability of the chick and this has become a well-cited example of

57 hormone-mediated adaptive maternal effects ${ }^{19-23}$.

58 The avian system of hatching asynchrony and maternal egg yolk hormones thus provides an

59 excellent study model for the interactions of two maternal effects. It also reveals a significant paradox

60 that has not yet been addressed adequately: if hatching asynchrony itself is an adaptive mechanism to

61 achieve efficient brood reduction by sibling competition depending on the amount of food available ${ }^{1,24}$,

62 why would mothers counteract this by increased androgen deposition in the later-laid eggs ${ }^{25,26}$ ?

As the research effort in this field grows rapidly, evidence that maternal androgens in egg

64 yolks do not always counteract the effects of hatching asynchrony has accumulated. An increasing

number of studies also found inconsistent effects from the experimental elevation of egg androgen concentrations, with sometimes opposite effects in the same species ${ }^{23}$. Rather than raising doubt about the adaptive explanations on within-clutch difference of maternal androgens, these inconsistent effects open up the possibility for an intriguing potential resolution to the HAAH paradox. Yolk androgen has been demonstrated to have both beneficial and detrimental effects. Thus, yolk androgens may have different effects depending on the food availability, and the androgens may act in concert with the effects of hatching asynchrony, which are also dependent on food availability. Egg androgens and hatching asynchrony together may then facilitate chick survival in good food conditions and promote brood reduction in poor food conditions.

75 it can also impose significant costs, such as increased resting metabolic rate in the short- and long-

76 term $^{27-29}$, suppressed immune function ${ }^{30-34}$ and oxidative damage ${ }^{35-39}$. We expect higher maternal

77 androgen to undermine survival in a year when resources are insufficient to offset its costs, but in a

78 year when food is abundant, higher maternal androgen exposure may help chicks obtain enough food

79 to offset the higher energy expenditure and the challenge to their immune system, and help them

80 survive.

81 We therefore hypothesize that androgens in eggs promote adaptive resource-dependent brood

82 reduction $^{26}$. If so, two pathways have co-evolved that allow mothers to facilitate resource-dependent 
83 brood reduction: in poor-food years, one pathway culls the brood to preserve offspring quality, and in

84 good-food years, the other pathway promotes survival to maximize offspring quantity. Combined,

85 these two pathways maximize the reproductive value of the brood in all food conditions. The key to

86 testing these resource-dependent effects of egg yolk androgens would be to combine a manipulation

87 of yolk androgens at the time of egg-laying, mimicking elevated maternal androgen deposition, and a

88 manipulation of food availability during the chick rearing phase, in a full factorial design. To our

89 knowledge, no such experiment has been conducted to test our hypothesis.

90 We performed this experiment in rock pigeons (Columba livia), an excellent species for our

91 study for two reasons: (1) its modal clutch size is two ${ }^{40}$ with the second egg always containing much

92 higher amounts of testosterone in the yolk than the first one $e^{41,42}$, and (2) it displays substantial

93 hatching asynchrony and the second chick is always smaller than the first one. In this study, we

94 created experimental clutches of first-laid eggs only, which contain low levels of maternal

95 testosterone. One egg was injected with testosterone solution to increase testosterone levels to those of

96 second-laid eggs, and the other was injected with vehicle as control. Both eggs were given to foster

97 parents and half of the resulting broods were reared in experimentally-imposed poor food conditions

98 while the other half were raised in good food conditions. Over the nestling period, we monitored

99 various aspects of growth and development as well as immune function and survival of the chicks. We

100 expected to see beneficial effects of elevated yolk testosterone on chick growth and immune function

101 under the good food condition, and detrimental effects under the poor food condition, specifically,

102 increased early mortality of chicks. 


\section{Materials and Methods}

We used pigeons from our rock pigeon colony housed in a large outdoor aviary. Before the experiment started (early April, 2012), breeding pairs of adult pigeons were re-housed in smaller identical aviaries, with two pairs in each aviary (see Supplementary Material for housing details). All experimental and animal care procedures were under the approval of the animal welfare committee of University of Groningen (DEC No. 5635D) and complied with Dutch law.

\section{Egg collection, incubation, hatching time and the creation of experimental broods}

In May (the annual peak of egg laying), we made the nest boxes available to induce breeding.

We checked nest boxes every morning and marked and collected all freshly-laid eggs, and replaced with a dummy egg so parents would start incubating. To increase the sensitivity of our experimental design, we aimed at creating experimental broods consisting of chicks of opposite treatments (testosterone or control injection) that were matched as much as possible in body mass, sex and hatching time. We only used first-laid eggs $\left(1^{\text {st }}\right.$ eggs $)$ in the experiment. Collected eggs were stored in a climate cell with relatively constant temperature $\left(12-16^{\circ} \mathrm{C}\right)$ and humidity $(40-50 \%)$ for no more than three days, until a large enough batch of eggs had been collected for creating experimental pairs. We paired eggs by matching laying date and mass as closely as possible, and then injected one egg in each pair with testosterone dissolved in sesame oil that increased levels in these $1^{\text {st }}$ eggs up to the level of the $2^{\text {nd }}$ eggs (testosterone eggs, see Egg injections in Supplementary Material) and injected the other with sesame oil only (control eggs). After injection, these eggs were returned immediately to an unrelated foster nest for incubation. The mass of testosterone-eggs and control-eggs did not differ significantly (mean \pm SD: testosterone-eggs, $17.00 \pm 1.27 \mathrm{~g}, \mathrm{n}=95$; control-eggs, $17.07 \pm 1.38 \mathrm{~g}, \mathrm{n}=95$; $\mathrm{t}=0.383, \mathrm{p}=0.7021$ ), nor laying date (Mann-Whitney $\mathrm{U}$ test, $\mathrm{p}=0.9779$ ).

On day 16 of incubation (about two days before hatching), all eggs were placed into an incubator until hatching and replaced in the nest by dummy eggs. The incubator was maintained at a constant $37.5{ }^{\circ} \mathrm{C}$ with humidity $>75 \%$. We checked the incubator every four hours between 9 am and $9 \mathrm{pm}$. We estimated hatching of hatchlings found at 9 am by the dryness of the down feathers as either 
at $3 \mathrm{am}$ (with relatively dry down) or at 7 am (with wetter down). We measured body mass, head-bill length, tarsus length, and wing length of each hatchling, and took a small blood sample $(<75 \mu l)$ from the medial metatarsal vein for sexing. We sexed the hatchlings with a molecular sexing procedure following the protocol described in Goerlich et al. $(2009,2010)^{41,43}$. We then matched hatchlings with opposite egg injection treatments in pairs by their hatching time and body mass, and returned paired hatchlings to unrelated foster nests. We successfully created 19 same-sex testosterone-control pairs of chicks with no significant difference in hatching time $(\mathrm{n}=29, \mathrm{t}=-1.79, \mathrm{p}=0.084)$ or body mass $(\mathrm{t}=-$ $1.229, \mathrm{p}=0.229$ ). Ten pairs were fostered by parents under good food conditions and 9 pairs by parents under poor food conditions.

\section{Food treatment}

We initiated the food treatment for parents on day 16 of incubation. Prior to the food treatment, a commercial seed mixture (KASPER ${ }^{\mathrm{TM}} 6721+6712$, Table S1), water, and a mixture of small stones and pigeon grit was available ad libitum to all pigeons. From the start of the food treatment onwards, pigeons in the good food condition group were also additionally provided with $a d$ libitum pigeon pellets (KASPAR ${ }^{\mathrm{TM}} \mathrm{P} 40$, Table $\mathrm{S} 1$ ) and supplemented with vitamin powder $\left(\right.$ Supralith $\left.^{\mathrm{TM}}\right)$. The food availability of the pigeons in poor food conditions was limited to $33 \mathrm{~g}$ of the seed mixture with broken corn kernels (lower protein and fat content, Table S1) per pair per day, the amount of average daily food consumption by a pair of adult pigeons according to our previous measurements. To accommodate increased energy demands as chicks grew, we provided the foodrestricted group with additional food when chicks were older than one week, based on Jacquin et al. $(2012)^{44}$. For every 7-day-old or older chick, we provided $8 \mathrm{~g}$ additional food; for every 14-day-old or older chick, $16 \mathrm{~g}$ of additional food was provided.

After the experiment, the body mass loss in adult pigeons in poor food conditions was significantly more than that of adults in good food conditions [good food condition, mean (\% of body mass before egg-laying) $\pm \mathrm{SE}=-6.31 \pm 0.75, \mathrm{n}=67$; poor food condition, mean $\pm \mathrm{SE}=-13.77 \pm 1.16, \mathrm{n}=45$, 
$156 \mathrm{t}=5.395, \mathrm{p}<0.0001]$, indicating that our experimental food restriction significantly reduced energy

157 intake to the adults.

\section{Chick monitoring}

Nests were checked every day to monitor chick survival. Four biometric variables: body mass,

head-bill length, tarsus length, and wing length were measured every two days in the first two weeks

after hatching, and every three days from two weeks to 26 days post-hatching. All body measurements samples were taken six days later. See supplementary material for details.

\section{Statistical analysis}

All statistical analyses were performed with the software R 3.5.2.

model to test the effects of treatment on hatching success, with hormone injection and egg mass as

predictors. We performed a Mann-Whitney U test to test for effects on hatching time, because it was

(GLM), including hormone injection, egg mass, and sex as predictors and also tested for sex-specific

effects of prenatal testosterone on hatchling body mass by including the interaction between hormone injection and sex.

We only tested the effects of treatment on chick body measurements until day 8 , because the sample size for testosterone chicks in the poor food condition after day 8 decreased drastically due to the high mortality in this group (see Results), yielding low statistical power and potential computational problems if analysing the whole growth curve. In addition, for chicks reared in good food conditions we also tested the effect of testosterone on each body measurement at day 26 , just before fledging. We used a general linear mixed model (R package $l m e 4)^{46}$, and Kenward-Roger approximation method to compute p-values (R package pbkrtest $)^{47}$. We included brood identity as a 
184 mass as a covariate, as it is known to strongly influence nestling body mass ${ }^{48}$. The interactions

185 between hormone and food treatment (for day 8 only), and between hormone treatment and sex (for

186 both day 8 and day 26) were also tested. Significant interactions were then further analysed with the

187 package phia $^{49}$ for pair-wise comparisons (Holm-adjusted p-values are presented). The results of the

188 four biometric variables showed clear consistency with each other, with effects on body mass being

189 the most pronounced. Therefore, only the results of growth in terms of body mass are reported below

190 and the other three (head-bill, tarsus and wing length) are reported in the supplementary materials.

191 For our analyses of immune response, we subtracted for each chick the pre-treatment score

192 from the final score of the SRBC-hemagglutination test. We applied a GLM to the resulting values,

193 with hormone injection, food treatment, and sex included as independent variables. The model

194 residuals did not violate assumptions of normality (Shapiro-Wilk test, $\mathrm{p}=0.492$ ). The interactions of

195 hormone injection with sex and hormone injection with food were also tested.

196 Chick survival was assessed between hatching to day 26, yielding right-censored data.

197 Kaplan-Meier survival curves for pigeons hatched from the testosterone-injected and control eggs and

198 reared under good and poor food conditions were built and tested using the package survival ${ }^{50}$ with

199 Gehan-Wilcoxon test and rho $=1$. 


\section{$200 \quad$ Results}

\section{Hatching success, hatching time, and hatchling body mass}

We injected a total of 190 eggs (95 testosterone-injected eggs and 95 control eggs) of which 93 eggs hatched successfully (48.95\%). Of those 93 eggs, 44 were control eggs and 49 were testosterone-injected eggs and this difference was not significant $(n=190, p=0.407)$. Heavier eggs were more likely to hatch successfully (estimate $\pm \mathrm{SE}=0.338 \pm 0.117, \mathrm{z}=2.895, \mathrm{p}=0.004$ ). There was no difference in time of hatching between testosterone-injected and control eggs (Mann-Whitney U test, $\mathrm{W}=1080, \mathrm{p}=0.991)$.

Of the 93 hatchlings, 90 had no visible developmental abnormalities and were included in the analysis of hatching body mass. Testosterone treatment did not affect hatchling body mass (marginal means \pm SE: hatchlings from testosterone-injected eggs, $11.58 \pm 0.11$; control hatchlings, $11.78 \pm 0.11$, $\mathrm{t}=-1.291, \mathrm{p}=0.200)$ and hatchling body mass was also not affected by sex (marginal means \pm SE: males, 11.66 \pm 0.11 ; females, $11.70 \pm 0.11, \mathrm{t}=-0.281, \mathrm{p}=0.780$ ) or the interaction between sex and hormone treatment $(\mathrm{p}=0.971)$. Only egg mass significantly predicted hatchling body mass (estimate $\pm \mathrm{SE}=$ $0.612 \pm 0.060, \mathrm{t}=10.152, \mathrm{p}<0.001)$.

\section{Chick survival}

Among the 90 hatchlings, we successfully created 19 same-sex testosterone-control pairs of chicks with 10 pairs reared under good food conditions and 9 pairs under poor food conditions. Their survival rates differed significantly among the four combinations of food treatment and testosterone injection ( $\mathrm{p}<0.001$, Fig. 1). In the good food conditions, chick survival was $100 \%(\mathrm{n}=20)$, regardless of hormone treatment, while in the poor food conditions, chicks from testosterone-injected eggs (hereafter "testosterone chicks") had lower survival than chicks from control eggs (hereafter "control chicks") $\operatorname{did}\left(\mathrm{n}=18, \chi^{2}=3.9, \mathrm{p}=0.049\right.$, Fig. 1). Overall, chicks reared under the poor food conditions had significantly lower survival than those under the good food conditions $\left(\mathrm{n}=38, \chi^{2}=12.8, \mathrm{p}<0.001\right)$. 
228 injection and food treatment was significant $\left(\mathrm{F}_{1,16.66}=6.758, \mathrm{p}=0.019\right.$, Fig. 2). Post-hoc interaction

229 analysis indicated that testosterone chicks were heavier (marginal mean $\pm \mathrm{SE}=120.49 \pm 4.42 \mathrm{~g}$ ) than

230 control chicks (marginal mean $\pm \mathrm{SE}=103.69 \pm 4.42 \mathrm{~g})$ in the good food conditions $\left(\chi^{2}=10.577\right.$, Holm-

231 adjusted $\mathrm{p}=0.002$ ), but not in the poor food conditions (marginal means $\pm \mathrm{SE}=55.74 \pm 4.69 \mathrm{~g}$ for

232 testosterone chicks; $58.66 \pm 4.63 \mathrm{~g}$ for control chicks, $\chi^{2}=0.277$, Holm-adjusted $\left.\mathrm{p}=0.599\right)$. The effect of

233 sex was not significant (marginal means \pm SE: males, $85.84 \pm 3.75 \mathrm{~g}$; females, $83.45 \pm 3.59 \mathrm{~g}$,

$\left.234 \mathrm{~F}_{1,15.32}=0.169, \mathrm{p}=0.687\right)$, nor was the interaction effect of testosterone treatment and sex $\left(\mathrm{F}_{1,16.40}=0.923\right.$, $235 \mathrm{p}=0.351)$.

236 For body mass at day 26, when chicks were about to fledge, we found that of the chicks

237 reared in the good food conditions, testosterone chicks were still on average $10.9 \mathrm{~g}(\mathrm{SE}=5.60)$ heavier

238 than control chicks, but the effect was not statistically significant $\left(\mathrm{F}_{1,8.55}=3.788, \mathrm{p}=0.085\right.$, Fig. 3).

239 Neither sex nor egg mass showed significant effects at this age ( $\mathrm{p}=0.312$ and 0.397 , respectively) and

240 there was no interaction between testosterone treatment and $\operatorname{sex}(\mathrm{p}=0.276)$.

\section{Immunocompetence}

Among the same-sex broods that survived to complete the SRBC tests $(n=28)$, the

244 testosterone fledglings showed significantly lower immune response against SRBC ( $\mathrm{n}=13$, marginal

245 mean $\pm \mathrm{SE}=2.05 \pm 0.75)$ than did control fledglings $(\mathrm{n}=15$, marginal mean $\pm \mathrm{SE}=4.08 \pm 0.68, \mathrm{t}=-2.030$,

$246 \mathrm{p}=0.041$, Fig. 4). Food restriction also showed a significant negative effect on the immune response

247 (marginal means $\pm \mathrm{SE}=5.18 \pm 0.56$ for fledglings reared under the good food conditions, $0.95 \pm 0.91$ for

248 fledglings reared under the poor food conditions, $\mathrm{t}=-4.230, \mathrm{p}<0.001$, Fig. 4). The interaction between

249 hormone and food treatment, however, did not have significant effects $(\mathrm{p}=0.396)$. There was no

250 significant sex difference $(\mathrm{p}=0.101)$ or interaction between hormone treatment and $\operatorname{sex}(\mathrm{p}=0.333)$. 


\section{Discussion}

Despite the fact that hatching asynchrony has been regarded as an adaptive maternal effect for optimizing the trade-off between offspring quantity and quality in different food conditions, maternal androgens in birds have been proposed as a tool for mothers to counteract the effects of hatching asynchrony ${ }^{18,19}$. This conceptual paradox, although put forward more than a decade ago ${ }^{19}$, still lacks sufficient effort to find a solution. The paradox could be resolved if maternal androgens have contextdependent effects, much like the context-dependent effects that hatching asynchrony has on survival of late-hatching offspring in good and poor food conditions. In this scenario, both maternal effects work together to downsize the brood when food is scarce by selectively hastening the death of the youngest offspring, and, when food is abundant, help rather than harm survival of late-hatching offspring by giving them, via maternal androgens, a boost in competing for food. Our findings support this idea: exposure to elevated yolk testosterone benefitted nestlings under good food conditions by enhancing growth (Fig. 2, 3), but caused higher mortality when food conditions were poor (Fig. 1). This is the first experimental evidence from a full factorial design that supports the notion of adaptive context-dependent effects of maternal hormones. It is also the first evidence that solves a long standing paradox in the field of hormone-mediated maternal effects, reconciling the seemingly opposing effects of hatching asynchrony and differential testosterone allocation to offspring. It shows that maternally engineered competitive asymmetries within broods arise from variation in yolk androgens and hatching asynchrony together, and allow parents to maximize the number of offspring they rear ${ }^{26}$. Moreover, in a system in which two maternal effects work in concert, mothers do not need to adjust the within-clutch pattern of testosterone deposition in relation to food abundance. As the effects of testosterone are context dependent, they can simply maintain higher testosterone concentrations in late-laid eggs regardless of pre egg-laying food conditions. This may explain why most studies did not find evidence for maternal adjustment of testosterone deposition in eggs in 
279 reported inconsistencies in the effects of in ovo injections of androgens ${ }^{23}$. Interestingly, the most cited

280 study for the detrimental effects of yolk testosterone on survival, the study on American kestrels

281 (Falco sparverius) ${ }^{51}$, was conducted in a poor food year (Sockman, personal communication). In contrast, a replication of that study on the closely related Eurasian kestrels (F. tinnunculus) in a good year found a positive effect (C. Dijkstra, J. Boonekamp and T.G.G. Groothuis, unpublished data). Similarly, a recently published field study on spotless starlings (Sturnus unicolor) reported that only among the second broods, when the food availability is generally deteriorated compared to when the parents raised their first broods, nestling mortality was significantly higher for the nestlings from androgen-treated eggs ${ }^{52}$. Another study by Cucco et al. $(2009)^{53}$, who injected different doses of testosterone into grey partridge (Perdix perdix) egg yolks and supplemented the diet with $\beta$-carotene in half of the chicks from each testosterone treatment, found that supplemented $\beta$-carotene can remedy the immunosuppressive effects induced by higher levels of yolk testosterone. It is therefore important that future studies testing the effect of yolk hormones take into account the food conditions. yolk testosterone, however, remains to have several unanswered questions. The enhancing effects of testosterone on growth in good food conditions may have been due to an increase in begging behaviour, as has been found in several studies ${ }^{54-56}$, which will result in higher food delivery by the parents. Furthermore, the food dependent effects of elevated maternal testosterone on growth may also have been mediated by increases in basal metabolic rate (BMR), as elevated prenatal testosterone exposure increases $\mathrm{BMR}^{27-29}$. Indeed, during food supplementation, increased BMR has been shown to enhance growth, whereas during food restriction, increased BMR can depress growth ${ }^{57,58}$. Therefore, the higher mortality of chicks from testosterone-injected eggs observed in the poor food condition may have been the result of a higher, but unsatisfied, energy demand. Our findings showed that most nestling mortality occurred after 8 days of age (Fig. 1). In this species, the energy and nutrient demands of chicks most likely peak around 10 days after hatching, and the higher energy demand around that time may have induced a fatally negative energy balance of testosterone-injected chicks in 
the poor food conditions. Moreover, if prenatal testosterone increased begging behaviour in the poor food conditions, this would have further exacerbated energy loss and the parents would not have been able to compensate it.

Our results indicate that regardless of food conditions, testosterone-injected fledglings showed a weaker response to an immune challenge (injected sheep red blood cells). This effect of immunosuppression is consistent with previous studies in other avian species ${ }^{30-34}$. Although the interaction effect on chicks' immune response between the yolk testosterone injection and food conditions was not significant, Figure 4 obviously suggests that a clear difference was only observed among chicks reared under good food conditions. This is likely due to a floor effect as food restriction induced already a very strong suppression of the immune response, and the suppressed responses would be difficult to differentiate as the antibody titers cannot go below 0 . This also suggests that high resource inputs are required to maintain good immune function ${ }^{59}$. It is possible that the high rate of energy expenditure caused by maternal testosterone exposure, combined with the low rates of energy inputs due to poor food conditions, left insufficient resources for maintaining the immune system.

In conclusion, our results suggest the co-evolution of two maternal effects to optimize the final brood size that are adaptive for the mother but not necessarily optimal for all offspring. The context dependent effects of maternally deposited testosterone on chick survival can be used as an explanation for the apparent discrepancies in the literature on this subject that currently hamper progress in our understanding to the functions of maternal hormones. When these are taken into consideration, the field of hormone-mediated maternal effects may move forward substantially.

\section{Acknowledgement}

We dedicate this paper to the memory of Dr. Cor Dijkstra, a beloved teacher, mentor, and friend, who peacefully left us in November, 2017. We greatly appreciate all animal care takers for their help, Roelie Veenstra-Wiegman for the help on sexing, Bernd Riedstra for the help on SRBC test, and Charlotte Deerenberg for valuable comments on the manuscript. 


\section{References}

333 1. Mock, D. W. \& Parker, G. A. The evolution of sibling rivalry. (Oxford University Press, New

$334 \quad$ York, 1997).

335 2. Roulin, A. \& Dreiss, A. N. Sibling competition and cooperation over parental care. In The

336 Evolution of Parental Care. (ed. Royle, N. J., Smiseth, P. T. \& Kölliker, M.) 133-149 (Oxford

337 University Press, 2012).

338 3. Frank, G. F., Glickman, S. E. \& Licht, P. Fatal sibling aggression, precocial development, and 339 androgens in neonatal spotted hyenas. Science 252, 702-704 (1991).

340 4. Hofer, H. \& East M. L. Siblicide in Serengeti spotted hyenas: a long-term study of maternal input 341 and cub survival. Behav. Ecol. Sociobiol. 62, 341-351 (2008).

342 5. Drummond, H. \& Garcia-Chavelas, C. Food shortage influences sibling aggression in the blue343 footed booby. Anim. Behav. 37, 806-819 (1989).

6. Stephenson, A. G. Flower and fruit abortion: proximate causes and ultimate functions. Annu. Rev. Ecol. Syst. 12, 253-279 (1981).

7. Hudson, R \& Trillmich, F. Sibling competition and cooperation in mammals: challenges, developments and prospects. Behav. Ecol. Sociobiol. 62, 299-307 (2008).

8. Andersen, I. L., Nævdal, E. \& Bøe, K. E. Maternal investment, sibling competition, and offspring survival with increasing litter size and parity in pigs (Sus scrofa). Behav. Ecol. Sociobiol. 65:

10.Groothuis, T. G. G. \& Schwabl, H. Hormone-mediated maternal effects in birds: mechanisms matter but what do we know of them? Phil. Trans. R. Soc. B 363, 1647-1661 (2008).

11.Carere, C. \& Balthazart, J. Sexual versus individual differentiation: the controversial role of avian maternal hormones. Trends Endocrinol. Metab. 18, 73-80 (2007).

Trans. R. Soc. 364, 1059-1074 (2009). 
359 13.Mousseau, T. A. \& Dingle, H. Maternal effects in insect life histories. Annu. Rev. Entomol. 36,

$360 \quad 511-534(1991)$.

361 14.Bowden, R. M., Ewert, M. A. \& Nelson C. E. Environmental sex determination in a reptile varies

362 seasonally and with yolk hormones. Proc. R. Soc. B 267, 1745-1749 (2000).

363 15.Schaafsma, S. M. \& Groothuis, T. G. G. Sex-specific effects of maternal testosterone on

364 lateralization in a cichlid fish. Anim. Behav. 83,437-443 (2012).

365 16.Cottrell, E. C. \& Seckl, J. R. Prenatal stress, glucocorticoids and the programming of adult disease.

$366 \quad$ Front. Behav. Neurosci 3, 19 (2009).

367 17.Dloniak, S. M., French, J. A. \& Holekamp, K. E. Rank-related maternal effects of androgens on

368 behavior in wild spotted hyaenas. Nature 440, 1190-1193.

369 18.Schwabl, H. Yolk is a source of maternal testosterone for developing birds. Proc. Natl. Acad. Sci.

$370 \quad$ U.S.A. 90, 11446-11450 (1993).

371 19.Groothuis, T. G. G., Müller, W., von Engelhardt, N., Carere, C. \& Eising, C. Maternal hormones

372 as a tool to adjust offspring phenotype in avian species. Neurosci. Biobehav. Rev. 29, 329-352

373 (2005).

374 20.Eising, C. M., Eikenaar, C., Schwabl, H., Groothuis, T. G. G. Maternal androgens in black-headed 375 gull (Larus ridibundus) eggs: consequences for chick development. Proc. R. Soc. Lond. B 268, $376 \quad 839-846(2001)$.

377 21.Müller, M. S., Roelofs, Y., Erikstad, K. E. \& Groothuis, T. G. G. Maternal androgens increase

378 sibling aggression, dominance, and competitive ability in the siblicidal black-legged

379 kittiwake (Rissa tridactyla). PLoS One 7, e47763 (2012).

380 22.Gil, D. Hormones in avian eggs: physiology, ecology and behavior. Adv. Study Behav. 38, 337-398

$381 \quad$ (2008).

382 23.von Engelhardt, N. \& Groothuis, T. G. G. Maternal Hormones in Avian Eggs. In Hormones and 383 Reproduction of Vertebrates. Vol. 4: Birds. (ed. Norris, D. O. \& Lopez, K. H.) 91-127 (Elsevier 384 Inc., Amsterdam, 2011). 
24.Amundsen, T. \& Slagsvold, T. Hatching asynchrony: facilitating adaptive or maladaptive brood reduction? Acta International Ornithological Congress, Christchurch, New Zealand XX: 1707$1719(1991)$.

25.Sockman, K. W., Sharp, P. J. \& Schwabl, H. Orchestration of avian reproductive effort: an integration of the ultimate and proximate bases for flexibility in clutch size, incubation behaviour, and yolk androgen deposition. Biol. Rev. 81, 629-666 (2006).

26.Müller, M. S. \& Groothuis, T. G. G. Within-clutch variation in yolk testosterone as an adaptive maternal effect to modulate avian sibling competition: evidence from a comparative study. Am. Nat. 181, 125-136 (2013).

27.Tobler, M., Nilsson, J.-Å. \& Nilsson, J. F. Costly steroids: egg testosterone modulates nestling metabolic rate in the zebra finch. Biol. Lett. 3, 408-410 (2007).

28.Nilsson, J. F., Tobler, M., Nilsson, J.-Å. \& Sandell, M.I. Long-lasting consequences of elevated yolk testosterone for metabolism in the zebra finch. Physiol. Biochem. Zool. 84, 287-291 (2011).

29.Ruuskanen, S. et al. Long-lasting effects of yolk androgens on phenotype in the pied flycatcher (Ficedula hypoleuca). Behav. Ecol. Sociobiol. 67, 361-372 (2013).

30.Andersson, S., Uller, T., Lõhmus, M. \& Sundtröm, F. Effects of egg yolk testosterone on growth and immunity in a precocial birds. J. Evol. Biol. 17, 501-505 (2004).

31.Groothuis, T. G. G., Eising, C. M., Dijkstra, C. \& Müller, W. Balancing between costs and benefits of maternal hormone deposition in avian eggs. Biol. Lett. 1, 78-81 (2005).

32.Müller, W. et al. Prenatal androgen exposure modulates cellular and humoral immune function of black-headed gull chicks. Proc. R. Soc. B 272, 1971-1977 (2005).

33.Navara, K. J., Hill, G. E. \& Mendonça, M. T. Variable effects of yolk androgens on growth, survival, and immunity in eastern bluebird nestlings. Physiol. Biochem. Zool. 78, 570-578 (2005).

34.Sandell, M. I., Tobler, M. \& Hasselquist, D. Yolk androgens and the development of avian immunity: an experiment in jackdaws (Corvus monedula). J. Exp. Biol. 212, 815-822 (2009).

35.Tobler, M. \& Sandell, M. I. Sex-specific effects of prenatal testosterone on nestling plasma antioxidant capacity in the zebra finch. J. Exp. Bio. 212, 89-94 (2009). 
412 36.Galván, I. \& Alonso-Alvarez, C. Yolk testosterone shapes the expression of a melanin-based

413 signal in great tits: an antioxidant-mediated mechanism? J. Exp. Biol. 213, 3127-3130 (2010).

414 37.Noguera, J. C., Alonso-Alvarez, C., Kim, S.-Y., Morales, J. \& Velando, A. Yolk testosterone

415 reduces oxidative damages during postnatal development. Biol. Lett. 7, 93-95 (2011).

38.Tobler, M., Sandell, M. I., Chiriac, S. \& Hasselquist, D. Effects of prenatal testosterone exposure on antioxidant status and bill color in adult zebra finches. Physiol. Biochem. Zool. 86, 333-345 (2013).

39.Treidel, L. A., Whitley, B. N., Benowitz-Fredericks, Z. M., Haussmann, M.F. Prenatal exposure to testosterone impairs oxidative damage repair efficiency in the domestic chicken (Gallus gallus).

Biol. Lett. 9, 20130684 (2013).

40.Johnston, R. E. \& Janiga, M. Feral Pigeons. (Oxford University Press, New York, 1995).

41.Goerlich, V. C., Dijkstra, C., Schaafsma, S. M. \& Groothuis, T. G. G. Testosterone has a long-term effect on primary sex ratio of first eggs in pigeons-in search of a mechanism. Gen. Comp. Endocrinol. 163, 184-192 (2009).

45.R Development Core Team. R: A language and environment for statistical computing. $\mathrm{R}$ 
47.Halekoh, U., Højsgaard, S. A Kenward-Roger approximation and parametric bootstrap methods for tests in linear mixed models - the R package pbkrtest. J. Stat. Softw. 59, 1-30 (2014).

48.Krist, M. Egg size and offspring quality: a meta-analysis in birds. Biol. Rev. 86, 692-716 (2011). 49.de Rosario-Martinez, H. phia: Post-hoc interaction analysis. R package version 0.2-1. (2015).

50.Therneau, T. A package for survival analysis in S. version 2.38. (2015).

51.Sockman, K. W. \& Schwabl, H. Yolk androgens reduce offspring survival. Proc. R. Soc. B 267, 1451-1456 (2000).

52.Muriel, J. et al. Context-dependent effects of yolk androgens on nestling growth and immune function in a multibrooded passerine. J. Evol. Biol. 28, 1476-1488 (2015).

53.Cucco, M., Guasco, B., Malacarne, G., Ottonelli, R. \& Tanvez, A. Yolk testosterone levels and dietary carotenoids influence growth and immunity of grey partridge chicks. Gen. Comp. Endocrinol. 156, 418-425 (2008).

54.Eising, C. M. \& Groothuis, T. G. G. Yolk androgens and begging behaviour in black-headed gull chicks: an experimental field study. Anim. Behav. 66, 1027-1034 (2003).

55.von Engelhardt, N., Carere, C., Dijkstra, C. \& Groothuis, T. G. G. Sex-specific effects of yolk testosterone on survival, begging, and growth of zebra finches. Proc. R. Soc. B 273, 65-70 (2006).

56.Noguera, J. C., Kim, S.-Y. \& Velando, A. Maternal testosterone influences a begging component that makes fathers work harder in chick provisioning. Horm. Behav. 64, 19-25 (2013).

57.Derting, T. L. Metabolism and food availability as regulators of production in juvenile cotton rats. Ecology 70, 587-595 (1989).

58.Auer, S. K, Salin, K., Rudolf, A. M., Anderson, G. J. \& Metcalfe, N.B. Flexibility in metabolic rate confers a growth advantage under changing food availability. J. Anim. Ecol. 84, 1405-1411 (2015).

59.Hasselquist, D. \& Nilsson, J.-Å. Physiological mechanisms mediating costs of immune responses: what can we learn from studies of birds? Anim. Behav. 83, 1303-1312 (2012). 


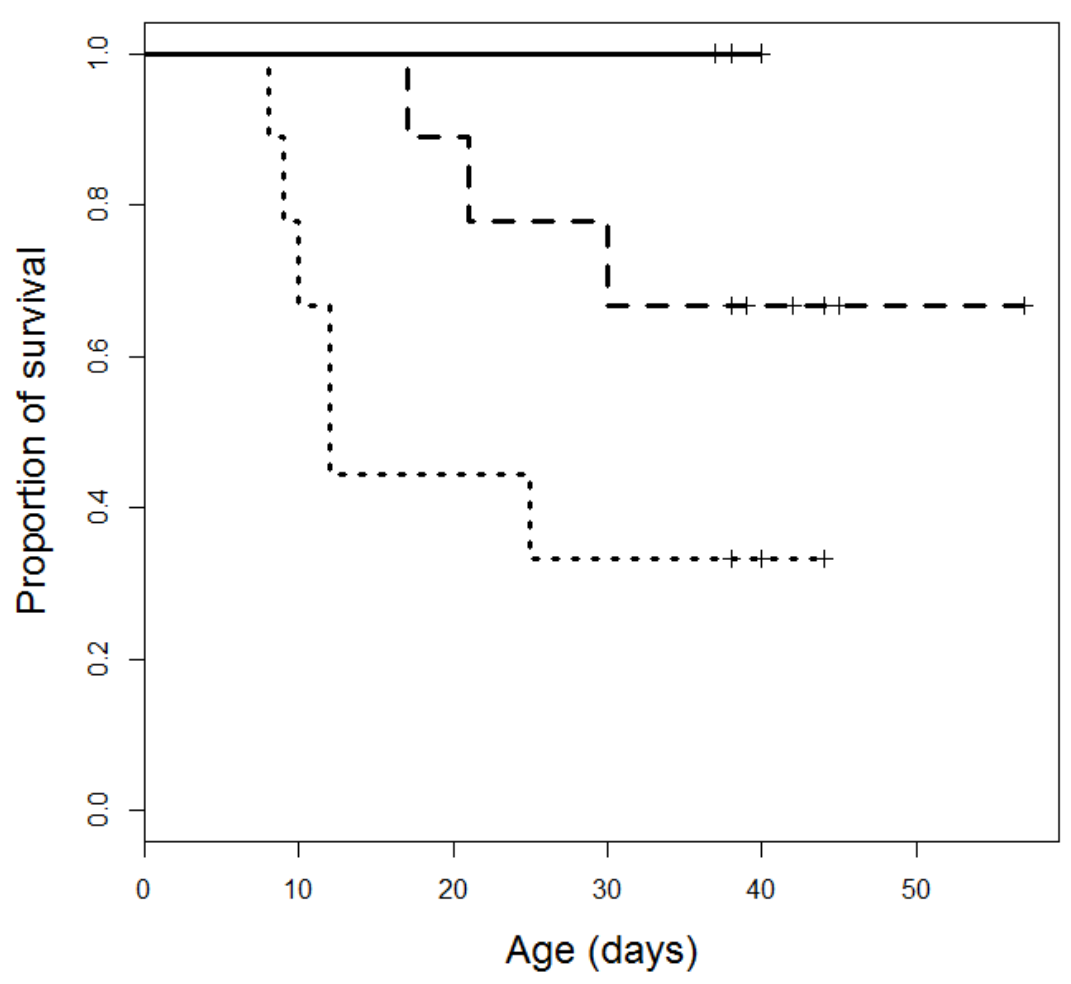

465 Figure 1. Survival curves of testosterone- and control-chicks. In good food conditions (solid line),

466 chicks from both egg injection treatment had 100\% survival so curves for testosterone- and control-

467 chicks overlap completely. In poor food conditions, Chicks from testosterone-injected eggs (dotted

468 line) had lower survival than control chicks (dashed line). 


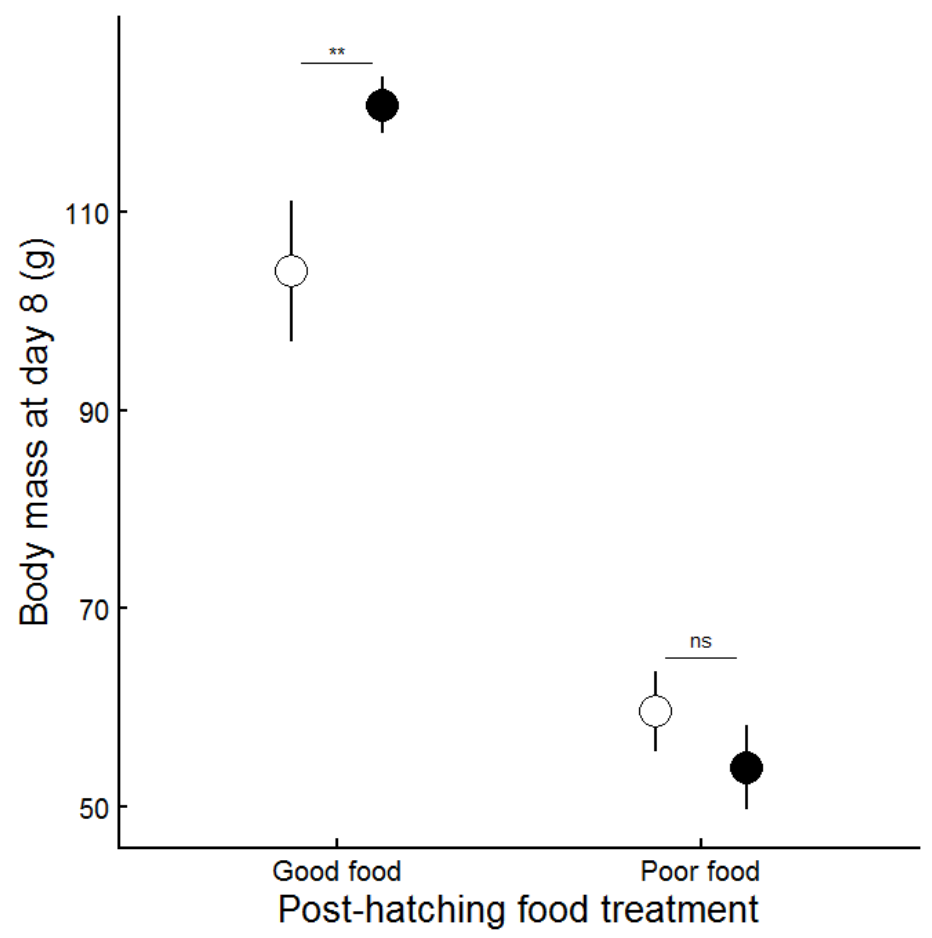

470 Figure 2. Means \pm SE of chick body mass at day 8 after hatching. Closed dots: chicks from

471 testosterone-injected eggs; open dots: control chicks from vehicle-injected eggs. Post-hoc analysis: **, $\mathrm{p}<0.01 ; \mathrm{ns}, \mathrm{p}>0.05$. 
bioRxiv preprint doi: https://doi.org/10.1101/2020.07.30.228528; this version posted July 31,2020. The copyright holder for this preprint (which was not certified by peer review) is the author/funder, who has granted bioRxiv a license to display the preprint in perpetuity. It is made available under aCC-BY-NC-ND 4.0 International license.

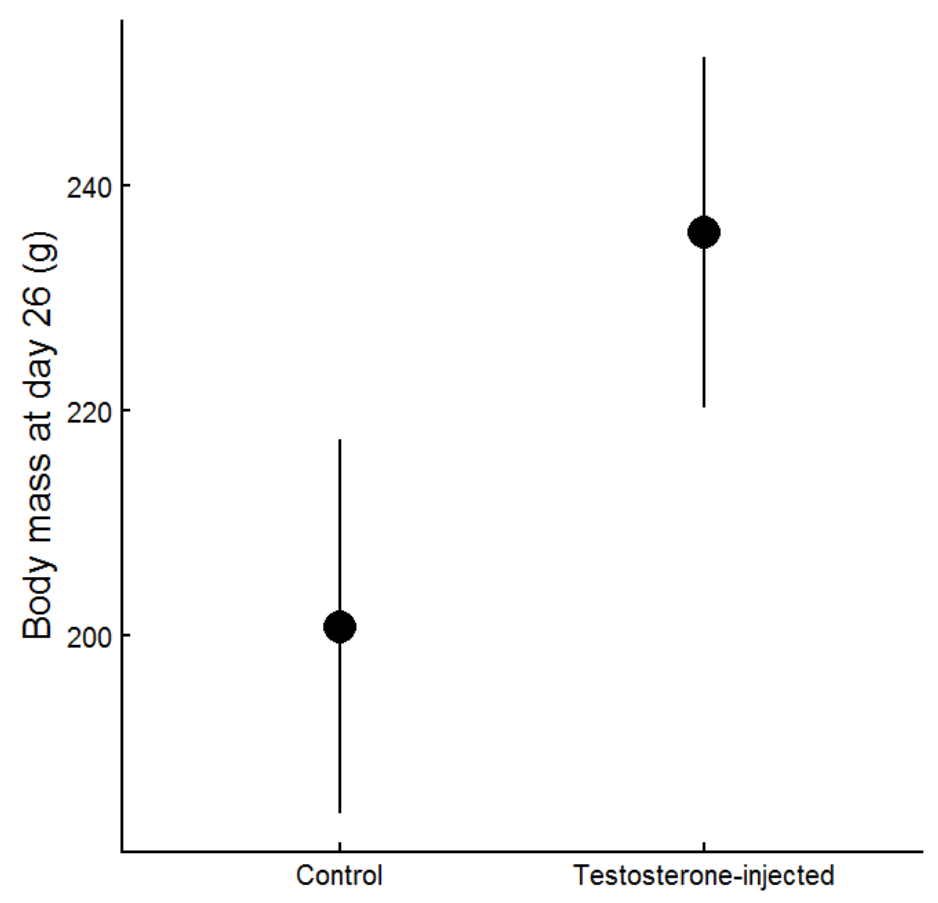

473

474 Figure 3. Mean \pm SE of chick body mass at day 26, around fledging. 
bioRxiv preprint doi: https://doi.org/10.1101/2020.07.30.228528; this version posted July 31, 2020. The copyright holder for this preprint (which was not certified by peer review) is the author/funder, who has granted bioRxiv a license to display the preprint in perpetuity. It is made available under aCC-BY-NC-ND 4.0 International license.

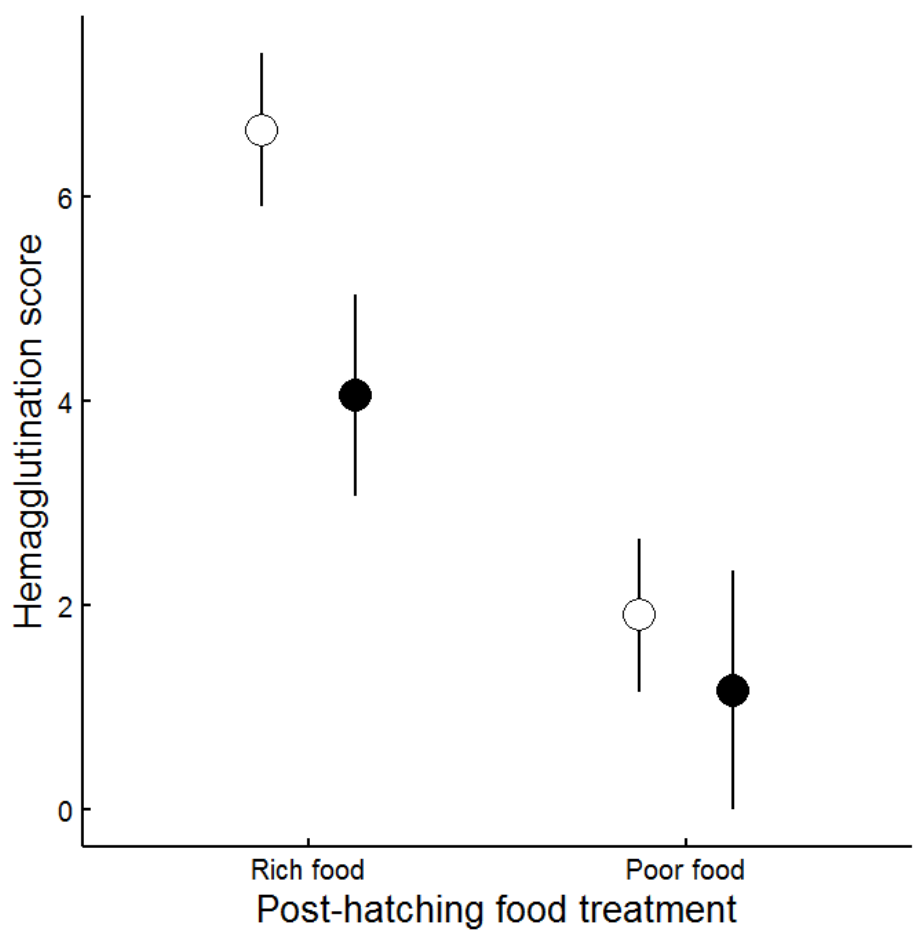

476 Figure 4. Mean \pm SE of the score of anti-SRBC antibody titres from hemagglutination assay. Closed

477 dots: chicks from testosterone-injected eggs; open dots: control chicks from vehicle-injected eggs. 